\title{
Gestión del Capital Intelectual y Desempeño de Grupos de Investigación Universitarios en un País Emergente. El caso de Colombia
}

\author{
Jose Arias-Pérez, Nelson Lozada* y Edwin Henao-García \\ Universidad de Antioquia, Facultad de Ciencias Económicas, Departamento de Admón. de Empresas, \\ calle 67 No. 53-108 oficina 13-414 Medellín-Colombia. (e-mail: nelson.lozada@udea.edu.co) \\ * Autor a quien debe dirigirse la correspondencia.
}

Recibido Nov. 21, 2018; Aceptado Ene. 24, 2019; Versión final Feb. 27, 2019, Publicado Ago. 2019

\begin{abstract}
Resumen
El objetivo del artículo es analizar la relación entre la gestión del capital intelectual y el desempeño de grupos de investigación universitarios. Se realizó un análisis clúster y de la varianza (ANOVA con comparaciones post-hoc) para identificar diferencias en medias significativas con datos obtenidos de una muestra de 288 grupos de investigación de universidades en Colombia. Los resultados ponen en evidencia la existencia de tres tipos de grupos: líderes, seguidores y rezagados. Entre ellos encontramos diferencias significativas en cuanto a la publicación de artículos científicos que tienen que ver con dos aspectos específicos de la gestión del capital intelectual: i) la evaluación sistemática de las competencias y el inventario de conocimientos del grupo, y ii) la existencia de una estrategia clara para desarrollar nuevas competencias y adquirir nuevos conocimientos. En conclusión, es necesario revaluar el enfoque tradicional de capital intelectual en universidades que limita la gestión de la construcción y monitoreo de indicadores.
\end{abstract}

\section{Intellectual Capital Management and Performance of University Research Groups in an Emerging Country, Colombia Case}

\begin{abstract}
The objective of the article is to analyze the relationship between the management of intellectual capital and the performance of university research groups (PURG). A cluster and variance analysis (ANOVA with posthoc comparisons) was performed to identify differences in significant means with data obtained from a sample of 288 university research groups in Colombia. The results show the existence of three types of groups: leaders, followers and laggards. We find significant differences in the publication of scientific articles among them that have to do with two specific aspects of the management of intellectual capital: i) the systematic evaluation of the competences and knowledge mapping of the group, and ii) the existence of a clear strategy to develop new skills and acquire new knowledge. In conclusion, it is necessary to rethink the traditional approach of intellectual capital for universities, which limits the construction and monitoring of performance indicators.
\end{abstract}

Keywords: intellectual capital management; research groups; performance indicators 


\section{INTRODUCCIÓN}

En los países emergentes la universidad sigue siendo el actor más importante en la construcción de la sociedad del conocimiento por ser la fuente de este recurso para los procesos de innovación y por ser el instrumento que tienen los ciudadanos para acceder al conocimiento científico-tecnológico (Arocena et al., 2015). Sin embargo la calidad de las universidades latinoamericanas sigue estando por debajo de los países desarrollados en cuanto a producción académica, número de doctores e inversión en investigación (OCDE, 2016). Los países latinoamericanos que ocupan las mejores posiciones en el escalafón de mejores universidades del mundo, son: Chile en el puesto 32, Brasil en el 41, Argentina en el puesto 45, Colombia en el 69 y México en el 87 (WEF, 2015). La literatura sugiere que el capital intelectual es uno de los enfoques para maximizar el uso de los activos de conocimiento de las universidades, bajo el entendido que inventariar recursos intangibles y generar indicadores de su existencia y evolución mejoran el desempeño de las universidades en materia de resultados de investigación (Cricelli et al., 2018). La mayoría de estudios se ha realizado en universidades europeas a partir de información secundaria, y se enfoca en generar metodologías para la construcción de indicadores de capital intelectual que tradicionalmente lo desagregan en capital humano, relacional y estructural (Ramírez et al., 2011; Ramírez y Gordillo, 2014; Secundo et al., 2015). En ese orden ideas, los resultados de investigación de las universidades dependen del inventario de capital humano que incluye número de doctores, de profesores de tiempo completo, entre otros aspectos; también, de capital estructural que alude a los recursos financieros, al número de artículos científicos, de programas académicos de pregrado y posgrado, y de grupos de investigación; y de capital relacional que incluye la movilidad estudiantil o profesoral, entre otros indicadores (Cricelli et al., 2018).

Lamentablemente, la discusión sobre capital intelectual en universidades se ha centrado en la construcción de ese tipo de indicadores, y en la construcción de metodologías para agregarlos y reportarlos, mientras se pasa por alto el componente de gestión del capital intelectual, que enfatiza la identificación y evaluación de conocimientos claves, y la planeación y el desarrollo de acciones orientadas a aumentar el inventario de los mismos (Kianto et al., 2013). Si el capital intelectual alude a los activos de conocimiento que son de propiedad de la organización, es decir, el inventario de conocimientos existentes en un momento determinado (Bontis, 2001), la gestión debe recaer de forma directa sobre esos activos intangibles, y no sólo de forma indirecta sobre indicadores como el número de doctores o de profesores de tiempo completo, como lo sugiere el enfoque tradicional en el contexto universitario. Por ende, este enfoque tradicional resulta bastante simplista porque limita la gestión al monitoreo de indicadores, a aspectos que por su naturaleza son más operativos que estratégicos, en lugar de darle mayor relevancia a la planeación y al despliegue de estrategias que dinamicen el flujo de conocimientos (Blanco et al., 2018). Esta lógica del enfoque tradicional también condiciona el alcance de los resultados de investigación porque los trabajos más importantes en torno a la relación entre capital intelectual y el desempeño investigativo de las universidades, se limitan a encontrar cuál de los indicadores mejora la producción científica, sin abrir la caja negra de la gestión. En ese sentido, el trabajo reciente más representativo que se ha realizado en el contexto de un país emergente, destaca que el tamaño de la universidad y la movilidad son los elementos asociados al capital intelectual que más incidencia tienen sobre el desempeño investigativo (Cricelli et al., 2018). Mientras que en países desarrollados se ha resaltado la importancia del capital estructural, particularmente de bases de datos, sistemas de información, entre otros (Bezhani, 2010; Andreeva y Garanina, 2016).

Sumado a ello, los trabajos sobre capital intelectual en el ámbito universitario obtienen sus datos principalmente de fuentes secundarias que son la base para la construcción de los reportes, sin embargo, recientemente se ha demostrado que estos indicadores tienen problemas de validez de contenido y no son la manera más adecuada para medir y poner en evidencia el capital intelectual (Altenburger y SchaffhauserLinzatti, 2015). Por ende, algunas preguntas de investigación sobre este constructo siguen sin resolverse por la ausencia de evidencia empírica concluyente. La investigación sobre capital intelectual en el ámbito universitario contrasta con la que se lleva a cabo en empresas, en donde se da mayor importancia a otros aspectos de la gestión como la planeación que a la construcción de indicadores y reportes, y a la realización de estudios basados en datos primarios, bajo el entendido que las mejoras en el desempeño organizacional a partir del capital intelectual se derivan de estrategias y acciones concretas que busquen identificar y aumentar el inventario de conocimientos claves (Kianto et al., 2013; Marzo y Scarpino, 2016), para lo cual los informes son insuficientes.

El objetivo del artículo es analizar la relación entre gestión del capital intelectual y el desempeño investigativo en universidades. Se realiza un análisis clúster y de la varianza (ANOVA con comparaciones post-hoc) para identificar las diferencias en medias significativas, con datos obtenidos de una muestra de 288 grupos de investigación de universidades públicas y privadas de Colombia, que por ley están obligadas a conformarlos por áreas de conocimiento, y sobre ellos recae la responsabilidad de producir conocimiento en ese país emergente (Robledo, 2007). 


\section{Gestión del capital intelectual}

El capital intelectual está relacionado con los activos de conocimiento que una organización posee, es decir, el inventario de conocimientos existentes en un momento determinado (Bontis, 2001), por ende la gestión del capital intelectual se concentra en el uso y la concepción estratégica de los procesos orientados a la consecución y explotación de activos de este tipo, para ello, este proceso comprende dos grandes actividades: la identificación y evaluación de conocimientos claves, y la planeación y desarrollo de acciones orientadas a aumentar el inventario de conocimientos (Kianto et al., 2013). La identificación de conocimientos claves comprende actividades que incluyen establecer qué, cómo y dónde se encuentra el conocimiento clave en una organización (Eppler, 2008; Balaid et al., 2016). Por su parte, la planeación y desarrollo de acciones implica la inclusión de procesos alusivos a la generación, adquisición, almacenamiento y diseminación del conocimiento (Ramírez et al., 2011). Esto implica también el desarrollo de mecanismos que permitan evaluar constantemente las actividades de planeación y estrategias utilizadas (Kianto et al., 2013).

\section{Desempeño de los grupos de investigación}

Las universidades en la gestión de sus ejes misionales, observándolo desde una perspectiva general, obtienen su desempeño en función de los resultados de la docencia, la investigación y la extensión, es decir en la generación y transferencia de conocimiento (Zangoueinezhad y Moshabaki, 2011). En ese marco, el propósito de este artículo es analizar el desempeño producto del eje misional de investigación el cual se materializa especialmente en la producción académica de artículos, literatura gris, entre otros aspectos (Abramo et al., 2014). En Colombia la política pública que orienta la investigación está asociada al Departamento Administrativo de Ciencia, Tecnología e Innovación, Colciencias, desde el cual se promueve la creación y consolidación de grupos de investigación asociados a las universidades los cuales desarrollan proyectos en torno a temáticas y líneas de investigación específicas, fruto de la madurez, fortalezas y el trabajo continuo que logran alcanzar las ciencias y disciplinas por parte de los académicos e investigadores al interior de las universidades. Es en el contexto de los grupos de investigación que las universidades materializan la producción investigativa, por esta razón tradicionalmente en la literatura se utiliza la expresión desempeño de grupos de investigación (Calderón et al., 2014; Robledo, 2007), que en el ámbito internacional normalmente se referencia como desempeño investigativo (Kyvik y Olsen, 2008).

\section{Gestión del capital intelectual y desempeño de los grupos de investigación}

Son múltiples las maneras en que la gestión del capital intelectual se relaciona e influye en el desempeño de grupos de investigación, una de éstas se asocia a la importancia que tiene para los grupos de investigación enfocar sus esfuerzos en conocimientos que aún no han sido suficientemente explotados y que a la vez tienen alto valor porque se consideran claves para la generación de nuevos conocimientos que como resultado natural se convierten en producción, es decir en desempeño. En este caso el proceso de identificación y el de evaluación de conocimientos son fundamentales (Lin et al., 2012; Kianto et al., 2013). Del mismo modo, la gestión del capital intelectual le facilita a los grupos el reconocimiento de vacíos de conocimiento, los cuales al explotarse son una fuente valiosa de productos académicos y científicos que jalonan y consolidan resultados de investigación sobresalientes (Graham et al., 2006).

La gestión del capital intelectual permite que los grupos de investigación dispongan de forma permanente de un diagnóstico de su inventario de conocimientos, a la vez que visualizan aquéllos que se requieren para mejorar el desempeño de grupos de investigación. En detalle, la gestión de activos intangibles implica que los grupos estén más activos e interesados en procesos enfocados a la generación, adquisición, almacenamiento y diseminación de conocimientos claves, como la construcción de redes interinstitucionales, la participación en eventos científicos y académicos, el intercambio de conocimiento, entre otras actividades (McFadyen y Cannella, 2004; Ramírez et al., 2011; Howell y Annansingh, 2013). Como resultado de la gestión del capital intelectual, el inventario de conocimientos claves robustecido produce una mejora de la producción académica y científica cuantificada en artículos, literatura gris, de los grupos de investigación (Ramírez et al., 2007; Cheng et al., 2009; Ramírez et al., 2014; Said et al., 2015 ; Calderón et al., 2017).

\section{METODOLOGÍA}

La hipótesis se contrastó en una muestra de grupos de investigación situados en Colombia, un país emergente ubicado en América Latina, rezagado en cuanto a inversión en ciencia y tecnología, que representa sólo el $0,2 \%$ del PIB, mientras que Brasil invierte el $1.2 \%$, Argentina el $0,6 \%$, México el $0.5 \%$ y Chile el $0,4 \%$, lo cual explica parcialmente su posición en el escalafón de las mejores universidades del mundo, que en la región lidera Chile en el puesto 32, Brasil en el 41, Argentina en el puesto 45, Colombia en el 69 y México en el 87 (WEF, 2015). Estas particularidades del contexto hacen más relevante la gestión del capital intelectual que constituye una estrategia para sortear las restricciones presupuestales de la investigación, y maximizar el 
aprovechamiento del inventario de conocimientos en términos de publicaciones. En el país hay 3000 grupos de investigación activos (OCyT, 2017); se logró estructurar una muestra de 288 que permiten una potencia de prueba satisfactoria, superior al $80 \%$ (Cohen, 1988). El trabajo de campo se realizó durante los meses de abril y mayo de 2016, mediante el envío del cuestionario por correo electrónico a integrantes de los grupos de investigación de universidades públicas y privadas del país. La tabla 1 indica la cantidad de grupos por área de conocimiento, la formación y cargo que ocupan los encuestados.

Tabla 1: Características de los grupos de investigación de la muestra

\begin{tabular}{|l|c|c|}
\hline \multicolumn{1}{|c|}{ Área del conocimiento } & Frecuencia & Porcentaje \\
\hline Ciencias sociales & 97 & 33,68 \\
\hline Ingeniería y tecnología & 74 & 25,69 \\
\hline Humanidades & 37 & 12,85 \\
\hline Ciencias agrarias & 20 & 6,94 \\
\hline Ciencias naturales & 29 & 10,07 \\
\hline $\begin{array}{l}\text { Ciencias médicas y de la } \\
\text { salud }\end{array}$ & 31 & 10,76 \\
\hline \multicolumn{1}{|c|}{ Total } & 288 & 100,00 \\
\hline \multicolumn{1}{|c|}{ Formación del encuestado } & Frecuencia & Porcentaje \\
\hline Doctorado & 131 & 45,49 \\
\hline Maestría & 116 & 40,28 \\
\hline Especialización & 18 & 6,25 \\
\hline Pregrado & 18 & 6,25 \\
\hline Otro & 5 & 1,73 \\
\hline \multicolumn{1}{c|}{ Total } & 288 & 100,00 \\
\hline Cargo del encuestado en el & Frecuencia & Porcentaje \\
\hline grupo de investigación & 109 & 37,85 \\
\hline Coordinador & 145 & 50,35 \\
\hline Investigador & 11 & 3,82 \\
\hline Estudiante en formación & 21 & 0,69 \\
\hline Asistente administrativo & 288 & 7,29 \\
\hline Otro & & \\
\hline & & \\
\hline
\end{tabular}

\section{Escalas de medida y fiabilidad}

Para la medición de la gestión del capital intelectual se emplearon los ítems propuestos por Kianto et al., (2013) y se utilizó una escala Likert que va de totalmente en desacuerdo (1) hasta totalmente de acuerdo (5). Para medir el desempeño de grupos de investigación se aplicó la escala propuesta por Ramayah et al. (2014) en lo que corresponde a la dimensión contribuciones escritas, con una escala Likert que va desde poco frecuente (1) hasta muy frecuente (5) (ver Tabla 2). Ambas escalas presentan un alfa de Cronbach superior a 0.7 , en el caso de gestión del capital intelectual es de 0.72 , y de 0.88 para el otro constructo, lo cual indica un nivel de fiabilidad satisfactorio (Hair et al., 2014).

Tabla 2: Escalas de medida

\begin{tabular}{|c|c|c|c|}
\hline Constructo & $\begin{array}{l}\text { Código } \\
\text { del ítem }\end{array}$ & Ítems & $\begin{array}{l}\text { Alfa de } \\
\text { Cronbach }\end{array}$ \\
\hline \multirow{3}{*}{$\begin{array}{l}\text { Gestión del } \\
\text { capital } \\
\text { intelectual }\end{array}$} & GCl1 & $\begin{array}{l}\text { El grupo tiene una visión clara sobre cuáles son actualmente sus saberes } \\
\text { o conocimientos más relevantes }\end{array}$ & \multirow{3}{*}{0.72} \\
\hline & $\mathrm{GCl}$ & $\begin{array}{l}\text { El grupo tiene una visión clara sobre cuáles son los conocimientos y } \\
\text { competencias necesarias para lograr sus objetivos }\end{array}$ & \\
\hline & GCl3 & $\begin{array}{l}\text { Se evalúan sistemáticamente las competencias y el inventario de saberes } \\
\text { o conocimientos del grupo }\end{array}$ & \\
\hline & $\mathrm{GCl} 4$ & $\begin{array}{l}\text { El grupo compara sus competencias y conocimientos más importantes } \\
\text { con los de otros grupos con los que existe alguna rivalidad por recursos o } \\
\text { reconocimiento }\end{array}$ & \\
\hline & GCI5 & $\begin{array}{l}\text { El grupo considera el desarrollo de nuevas competencias y conocimientos } \\
\text { como un factor clave en los ejercicios de planeación }\end{array}$ & \\
\hline & GCI6 & $\begin{array}{l}\text { El grupo tiene una estrategia clara para desarrollar nuevas competencias } \\
\text { y adquirir nuevos saberes o conocimientos }\end{array}$ & \\
\hline
\end{tabular}


Tabla 2 (continuación)

\begin{tabular}{|l|c|l|c|}
\hline \multirow{4}{*}{$\begin{array}{c}\text { Desempeño de } \\
\text { los grupos de } \\
\text { investigación }\end{array}$} & DGl1 & Publicar artículos en revistas científicas y en otros medios de divulgación & \\
\cline { 2 - 5 } & DGl3 & $\begin{array}{l}\text { Compartir archivos personales relacionados con investigación en } \\
\text { desarrollo }\end{array}$ & $\begin{array}{l}\text { Generar opinión escrita que aporte a las discusiones que se promueven } \\
\text { en su facultad }\end{array}$ \\
\cline { 2 - 5 } & DG14 & $\begin{array}{l}\text { Divulgar información relacionada con la universidad en espacios de } \\
\text { discusión virtuales }\end{array}$ & \\
\hline
\end{tabular}

Análisis de los datos

En primer lugar se realizó un análisis clúster jerárquico, se aplicó el método de Ward, para identificar grupos con características similares en cuanto a gestión de capital intelectual y desempeño de grupos de investigación. Posteriormente se realizó un análisis de la varianza (ANOVA) que implica la realización de la prueba de Levene para determinar si existe homogeneidad de varianzas y la verificación de la significancia del estadístico $\mathrm{F}$, que es el que permite establecer si las diferencias en medias son significativas entre los tres grupos (Merino y Diaz, 2006).

Luego se realizaron comparaciones post-hoc para establecer de manera específica entre cuáles de los tres grupos se presentan las diferencias significativas, se aplicó el procedimiento Tukey para el factor que cumpla el supuesto de las varianzas iguales, y el Games-Howell cuando se presenta un incumplimiento del mismo. En ambos casos se procedió a verificar si las medias de cada grupo en particular difiere del nivel de significación del 0.05 (Merino y Diaz, 2006).

\section{RESULTADOS Y DISCUSIÓN}

En primer lugar en la muestra de 288 grupos se identificaron tres grupos con características similares en cuanto a gestión de capital intelectual y desempeño de grupos de investigación. A los que presentan las puntuaciones más altas en todos los ítems se les ha denominado líderes $(\mathrm{N}=117)$, los que se encuentran en un punto intermedio son los seguidores $(\mathrm{N}=128)$, y los que están por debajo de los anteriores son los rezagados $(\mathrm{N}=43)$.

En cuanto a la gestión de capital intelectual y desempeño de grupos de investigación sólo en las variables GCl3 y GCl6 se cumple el supuesto de la homogeneidad de la varianza, lo cual indica que son significativas las diferencias en medias entre los grupos, dado que el valor del nivel crítico (Sig.) del estadístico $\mathrm{F}$ se encuentra por debajo de 0.05; La diferencia de medias es significativa al nivel ,05 ( ${ }^{*}$ Post-Hoc: Tukey), (ver Tabla 3). Sumado a ello, las comparaciones post-hoc permitieron establecer que son significativas, entre cada uno de los tres grupos, las diferencias en materia de evaluación sistemática de las competencias y del inventario de saberes o conocimientos del grupo, y en cuanto a la claridad de la estrategia para desarrollar nuevas competencias y adquirir nuevos conocimientos.

Tabla 3: Diferencias en medias significativas en cuanto a gestión del capital intelectual

\begin{tabular}{|c|c|c|c|c|c|c|c|}
\hline \multirow[b]{2}{*}{ Variables } & \multicolumn{3}{|c|}{ Media de los clústeres } & \multicolumn{2}{|c|}{ Estadístico igualdad de varianzas } & \multicolumn{2}{|c|}{ ANOVA } \\
\hline & $\begin{array}{l}\text { Líderes } \\
(N=117)\end{array}$ & $\begin{array}{c}\text { Seguidores } \\
(N=128)\end{array}$ & $\begin{array}{c}\text { Rezagados } \\
(N=43)\end{array}$ & Levene & Sig. & $F$ & Sig. \\
\hline $\mathrm{GCl} 1$ & 4.56 & 4.23 & 2.49 & 15,359 &, 000 & 155,384 &, 000 \\
\hline $\mathrm{GCl} 2$ & 4.58 & 4.23 & 2.42 & 16,533 & ,000 & 166,665 &, 000 \\
\hline $\mathrm{GCl} 3$ & $4.09^{*}$ & $3.06^{*}$ & $1.58^{*}$ & ,623 &, 537 & 138,343 &, 000 \\
\hline $\mathrm{GCl} 4$ & 3.39 & 2.53 & 1.65 & 4,906 & ,008 & 50,035 &, 000 \\
\hline GCl5 & 4.32 & 3.83 & 2.09 & 11,003 &, 000 & 106,973 &, 000 \\
\hline GCI6 & $4.13^{*}$ & $3.37^{*}$ & $1.53^{*}$ & , 149 & ,862 & 150,977 &, 000 \\
\hline
\end{tabular}

En cuanto al desempeño de grupos de investigación el valor del nivel crítico (Sig.) sugiere que las medias son significativas, pero la prueba de Levene indica que no se cumple el supuesto de igualdad de la varianza, sin embargo la comparación post-hoc que procede en este caso indica que hay diferencias en todas las variables del DGI. En el caso de la variable DGl1 relacionada con la publicación de artículos científicos, las diferencias son significativas entre todos los grupos entre sí, pero en las demás variables sólo es significativa entre los líderes y los otros dos grupos, pero no entre seguidores y rezagados. La diferencia de medias es significativa al nivel ,05 ('Post-Hoc: Games- Howell), (ver tabla 4). 
Tabla 4: Diferencias en medias significativas en cuanto a desempeño de grupos de investigación

\begin{tabular}{|c|c|c|c|c|c|c|c|}
\hline \multirow[b]{2}{*}{ Variables } & \multicolumn{3}{|c|}{ Media de los clústeres } & \multicolumn{2}{|c|}{ Estadístico igualdad de varianzas } & \multicolumn{2}{|c|}{ ANOVA } \\
\hline & $\begin{array}{l}\text { Lideres } \\
(N=117)\end{array}$ & $\begin{array}{c}\text { Seguidores } \\
\quad(N=128)\end{array}$ & $\begin{array}{c}\text { Rezagados } \\
\quad(N=43)\end{array}$ & Levene & Sig. & $F$ & Sig. \\
\hline DGl1 & $3.79^{*}$ & $3.24^{*}$ & $2.60^{*}$ & 4,958 & ,008 & 17,396 &, 000 \\
\hline DGI2 & $3.85^{*}$ & 2.34 & 2.26 & 4,343 & 014 & 71,251 &, 000 \\
\hline DGI3 & $3.79^{*}$ & 1.99 & 2.00 & 6,472 & ,002 & 115,326 &, 000 \\
\hline DGI4 & $3.63^{*}$ & 1.89 & 2.12 & 11,381 &, 000 & 101,129 &, 000 \\
\hline
\end{tabular}

Estos resultados indican que aspectos específicos de la gestión del capital intelectual relacionados con la evaluación sistemática de las competencias y del inventario de saberes o conocimientos del grupo, y con la claridad de la estrategia para desarrollar nuevas competencias y adquirir nuevos conocimientos, son los que explican la ventaja de los grupos líderes en materia de publicación de artículos científicos, generación de opinión escrita, y demás aspectos del desempeño de grupos de investigación. Así mismo los resultados del estudio muestran con claridad que los grupos de investigación, que diagnostican sistemáticamente su capital intelectual y tienen una estrategia para desarrollar y adquirir nuevos conocimientos y competencias, son los que obtienen los mejores resultados investigativos, particularmente en cuanto a la publicación de artículos científicos en donde son más notorias las diferencias. Llama la atención que aspectos de la gestión del capital intelectual como la identificación de conocimientos claves o la comparación de competencias con otros grupos con los que puede haber una rivalidad, son actividades en las que no hay diferencias significativas, probablemente porque son aspectos que pueden ser de carácter enunciativo, están en el plano estrictamente analítico y carecen de hoja de ruta para idear mejoras del inventario de conocimiento. Sin embargo, las diferencias aparecen en los ítems evaluación y estrategia de adquisición de nuevos conocimiento, que por su naturaleza son componentes de la gestión del capital intelectual más orientados a la puesta en marcha de acciones concretas, que a la larga son los que explican la diferencia en publicación de artículos científicos entre los grupos.

Estos resultados contrastan con estudios previos realizados en países emergentes y desarrollados, en países emergentes se ha destacado el tamaño de la universidad y la movilidad de estudiantes y profesores como los elementos asociados al capital intelectual que más incidencia tiene sobre el desempeño investigativo (Cricelli et al., 2018), mientras que en países desarrollados se ha resaltado la importancia del capital estructural, particularmente de bases de datos, sistemas de información, entre otros (Bezhani, 2010; Andreeva y Garanina, 2016). Este contraste obedece a que los estudios que se han hecho hasta el momento en el ámbito universitario que se analizan la incidencia directa de los activos de conocimiento sobre los resultados en investigación (Cricelli et al., 2018), se concentran en los indicadores de capital intelectual, pero no consideran de forma específica la gestión de ese inventario como se ha planteado en el presente artículo. En otras palabras, el enfoque tradicional no aborda de forma directa los activos intangibles o conocimientos que son la columna vertebral del concepto de capital intelectual, lo hace de forma indirecta construyendo indicadores a partir de elementos tangibles, por ejemplo una base de datos como indicador de capital estructural (Andreeva y Garanina, 2016). De este modo, el enfoque tradicional asume que a mayor cantidad de bases de datos es mayor el inventario de activos intangibles, por ende, la gestión del capital intelectual termina recayendo no sólo sobre este tipo de indicador, sino sobre artefactos organizacionales. En consecuencia, paradójicamente los intangibles que son la principal fuente de ventaja competitiva de los grupos de investigativos, suelen estar por fuera del radar de este proceso de gestión, por ejemplo, una metodología de investigación, una nueva técnica estadística, un protocolo de intervención, un modelo de consultoría, entre otros. De ahí la importancia del presente artículo que intenta abordar de forma directa los intangibles, mediante su identificación y evaluación entre otros componentes implícitos en este tipo de gestión.

\section{ANÁLISIS FINAL}

El análisis de los resultados obtenidos en el estudio permite establecer como principales cuatro elementos:

1) La importancia de adopción del enfoque de gestión de capital intelectual en el contexto universitario, ampliamente utilizado en el contexto empresarial, en momentos en los que el enfoque tradicional se concentra en la construcción de indicadores, cuya validez de contenido ha quedado en entredicho recientemente y hay dudas en torno a la conveniencia de usar información secundaria para poner en evidencia aspectos del capital intelectual (Altenburger y Schaffhauser-Linzatti, 2015).

2) En consecuencia, los resultados de este estudio obligan a reorientar esta discusión y a profundizar la utilización de este enfoque en este campo de estudio, que permite la medición de la gestión de capital intelectual con base en información primaria y con escalas que presentan unos indicadores altos de fiabilidad 
y validez, lo cual permitiría superar esta limitación medular del enfoque tradicional permitiendo aproximaciones más adecuadas a este objeto de estudio.

3) Las universidades deberían superar el enfoque actual de gestión que prioriza la construcción de reportes e indicadores con propósitos estrictamente informativos, y concentrarse en asuntos más específicos que pasan inadvertidos, como el desarrollo de la estrategia para que un grupo de investigación logre dominar una determinada metodología que es relevante en su campo de estudio, que de ser incorporada al inventario de conocimientos podría mejorar de forma substancial su producción de artículos científicos y demás indicadores de desempeño de grupos de investigación. En otras palabras, la gestión debe recaer de forma directa sobre esos activos intangibles, y no sólo de forma indirecta sobre indicadores como el número de doctores o de profesores de tiempo completo, como lo sugiere el enfoque tradicional de estudio del capital intelectual en el contexto universitario.

4) Los resultados se restringen al ámbito universitario de un país emergente, que comparte con sus pares latinoamericanos un rezago en contraste con universidades de países desarrollados en lo referente a producción de conocimiento, número de doctores e inversión en investigación, donde la gestión del capital intelectual constituye una estrategia para maximizar el aprovechamiento del inventario de conocimiento; por esta razón existen limitaciones para extrapolar los resultados a las universidades que no están supeditadas a este tipo de restricciones y que ocupan las primeras posiciones en los ránquines de calidad.

\section{CONCLUSIONES}

El análisis de los resultados obtenidos en el estudio permite establecer como principales conclusiones:

1) La acumulación de activos de conocimiento de manera desarticulada y sin ninguna consideración estratégica resulta insuficiente para la obtención de ventajas competitivas y de mejoras en el desempeño de los grupos de investigación.

2) Los grupos de investigación que diagnostican sistemáticamente su capital intelectual y tienen una estrategia para desarrollar y adquirir nuevos conocimientos y competencias, son los que obtienen el mejor DGI.

3) La prevalencia de estudios basados en datos secundarios impone una serie de restricciones metodológicas, que probablemente son las que explican por qué los trabajos sobre $\mathrm{Cl}$ en el ámbito universitario son repetitivos en torno al desarrollo de metodologías, para la construcción de indicadores e informes de $\mathrm{Cl}$ en detrimento de otros aspectos que demandan más investigación como la GCl.

\section{AGRADECIMIENTOS}

Este artículo hace parte de los productos asociados al proyecto de investigación denominado: "Efecto de la co-creación de valor y la co-innovación sobre el desempeño innovador en las PYMES de Medellín-Antioquia", dicho proyecto se incluye en la estrategia de financiamiento a la investigación por parte del CODI -Comité para el Desarrollo de la Investigación- de la Universidad de Antioquia, la ejecución del proyecto se extendió durante los años 2017-2018.

\section{REFERENCIAS}

Abramo, G., C. D'Angelo y F. Rosati, Career advancement and scientific performance in universities, doi: 10.1007/s11192013-1075-8, Scientometrics, 98(2), 891-907 (2014)

Altenburger, O. y M. Schaffhauser-Linzatti, The suitability of intellectual capital reports for the quantitative measurement of overall university performance, Incentives and performance, 379-396, Springer, Cham (2015)

Andreeva, T. y T. Garanina, Do all elements of intellectual capital matter for organizational performance? Evidence from Russian context, Journal of Intellectual Capital, 17(2), 397-412 (2016)

Arocena, R., B. Göransson y J. Sutz, Knowledge policies and universities in developing countries: Inclusive development and the developmental university, doi: 10.1016/j.techsoc.2014.10.004, Technology in society, 41, 10-20 (2015)

Balaid, A., M. Abd, S. Hikmi y J. Memon, Knowledge maps: A systematic literature review and directions for future research, doi: 10.1016/j.jijnfomgt.2016.02.005, International Journal of Information Management, 36(3), 451-475 (2016)

Bezhani, I., Intellectual capital reporting at UK universities, doi: 10.1108/14691931011039679, Journal of Intellectual Capital, 11(2), 179-207 (2010)

Blanco-Valbuena, C., C. Bernal-Torres, F. Camacho y M. Díaz-Olaya, Industrias creativas y culturales: estudio desde el enfoque de la gestión del conocimiento, doi: 10.4067/S0718-07642018000300015, Información Tecnológica, 29(3), $15-28(2018)$ 
Bontis, N., Assessing knowledge assets: a review of the models used to measure intellectual capital, doi: 10.1111/14682370.00053, International Journal of Management Reviews, 3(1), 41-60 (2001)

Calderón, G., G. Castaño y otros cuatro autores, Generación de conocimiento en los grupos élite de investigación en administración en Colombia, 1를. Ed., Editorial Universidad Nacional de Colombia, Bogotá, Colombia (2014)

Calderón, G., Á. Aristizabal y otros ocho autores, La generación de conocimiento en estrategia organizacional en Colombia, 1르. Ed., Universidad Sergio Arboleda - Asociación Colombiana de Facultades de Administración, Bogotá, Colombia (2017)

Cheng, M., J. Ho y P. Lau, Knowledge Sharing in Academic Institutions: a Study of Multimedia University Malaysia, Electronic Journal of Knowledge Management, 7(3), 313-324 (2009)

Cohen, J., Statistical power analysis for behavioral sciences, Academic Press, New York, Estados Unidos (1988)

Cricelli, L., M. Greco, M. Grimaldi y L. Llanes, Intellectual capital and university performance in emerging countries: evidence from Colombian public universities, doi: 10.1108/JIC-02-2017-0037, Journal of Intellectual Capital, 19(1), 7195 (2018)

Eppler, M., A process-based classification of knowledge maps and application examples, doi: 10.1002/kpm.299, Knowledge and Process Management, 15(1), 59-71 (2008)

Graham, I., J. Logan y otros cinco autores, Lost in knowledge translation: time for a map?, doi: 10.1002/chp.47, The Journal of Continuing education in the health professions, 26(1), 13-24 (2006)

Hair, J., T. Hult, C. Ringle y M. Sarstedt, A primer on partial least squares structural equation modeling (PLS-SEM), Sage, California, EUA (2014)

Howell, K. y F. Annansingh, Knowledge generation and sharing in UK universities: A tale of two cultures? doi: 10.1016/j.ijinfomgt.2012.05.003, International Journal of Information Management, 33(1), 32-39 (2013)

Kianto, A., T. Andreeva e Y. Pavlov, The impact of intellectual capital management on company competitiveness and financial performance, doi: 10.1057/kmrp.2013.9, Knowledge Management Research \& Practice, 11(2), 112-122 (2013)

Kyvik, S. y T. Olsen, Does the aging of tenured academic staff affect the research performance of universities? doi: 10.1007/s11192-007-1767-z, Scientometrics, 76(3), 439-455 (2008)

Lin, C., L. Edvinsson, J. Chen y T. Beding, National intellectual capital and the financial crisis in Brazil, Russia, India, China, Korea and South Africa, Springer, New York, EUA (2012)

Marzo, G. y E. Scarpino, Exploring intellectual capital management in SMEs: an in-depth Italian case study, doi: 10.1108/JIC-09-2015-0075, Journal of Intellectual Capital, 17(1), 27-51(2016)

McFadyen, A. y A. Cannella, Social capital and knowledge creation: Diminishing returns of the number and strength of exchange relationships, doi: 10.2307/20159615, Academy of Management Journal, 47(5), 735-746 (2004)

Merino, A. y M. Ruiz, Análisis de datos con SPSS 13 Base, McGraw-Hill Interamericana, Madrid, España (2006)

OCDE, Fomentando un crecimiento inclusivo de la productividad en América Latina, OCDE, París, Francia (2016)

OCYT, Indicadores de ciencia y tecnología Colombia 2016, Observatorio Colombiano de Ciencia y Tecnología, Bogotá, Colombia (2017)

Ramayah, T., J. Yeap y J. Ignatius, Assessing Knowledge Sharing among Academics: A Validation of the Knowledge Sharing Behavior Scale (KSBS), doi: 10.1177/0193841X14539685, Evaluation Review, 38(2), 160-187 (2014)

Ramírez, Y., C. Lorduy y J. Rojas, Intellectual capital management in Spanish universities, doi: 10.1108/14691930710830873, Journal of Intellectual Capital, 8 (4), 732-748 (2007)

Ramírez, Y. y S. Gordillo, Recognition and measurement of intellectual capital in Spanish universities, doi: 10.1108/JIC05-2013-0058, Journal of Intellectual Capital, 15(1), 173-188 (2014)

Ramírez, Y., J. Santos y Á. Tejada, Intellectual capital in Spanish public universities: stakeholders' information needs, doi: 10.1108/14691931111154689, Journal of Intellectual capital, 12(3), 356-376 (2011)

Robledo, J., From consolidated research groups to dynamic innovation systems: the present challenge of the Colombian scientific and technological development, DYNA, 74(152), 1-7 (2007)

Said, A., R. Rasdi y otros tres autores, A career success model for academics at Malaysian research universities, European Journal of Training and Development, doi: 10.1108/EJTD-03-2015-0022, 39(9), 815-835 (2015)

Secundo, G., S. Elena-Perez, Ž. Martinaitis y K. Leitner, An intellectual capital maturity model (ICMM) to improve strategic management in european universities: a dynamic approach, doi: 10.1108/JIC-06-2014-0072, Journal of Intellectual Capital, 16(2), 419-442 (2015)

WEF, The Global Competitiveness Report 2014-2015. World Economic Forum, Berna, Suiza (2015) 